\title{
Reliability of Arch Retaining Structure with Stochastic Parameters
}

\author{
Liu Ming ${ }^{*}, 1$, Wu Yongping ${ }^{2}$ and Qiao Xinzhou ${ }^{2}$ \\ ${ }^{I}$ College of Sciences, Xi'an University of Science and Technology, Xi'an, Shaanxi, 710054, P.R. China \\ ${ }^{2}$ School of Mechanical Engineering, Xi'an University of Science and Technology, Xi'an, Shaanxi, 710054, P.R. China
}

\begin{abstract}
Aimed at the reliability problems of arch retaining structure with stochastic parameters, in consideration of the influences of the randomness of the structural parameters and loads, the concrete paper applies program building relevant arch retaining structural model. The simulation programs are based on the response surface method and Monte Carlo method hybrid simulation analysis method. The structure failure probability and the probability distribution of the maximum stress of arch retaining structure are obtained in this study: get the influence of different random parameters on the structural reliability of arch retaining structure. This method can offer a theoretical basis for roadway supporting structure analysis and design.
\end{abstract}

Keywords: Arch retaining structure, randomness, reliability, stochastic parameter.

\section{INTRODUCTION}

Due to the complex environment with many uncertainty factors in soft rock roadway, supporting structure design method of the traditional reliability [1-10] is low and cannot fully meet the needs of the project. This uncertainty is mainly: (1) the load environment of surrounding rock are uncertain, especially on the theory of tectonic stress is still very incomplete. (2) the uncertainty surrounding environment, including the discreteness of rock strength index, the heterogeneity of rock mass, discontinuousness, nonlinearity, nonisotropy and so on. (3) the material uncertainty of roadway support structure.

Analysis and design for roadway supporting structure, traditional calculation methods are using deterministic model, without considering the uncertainty of the load and structure, and other multi-factor, So that it makes a big difference between the calculation results and the actual results. The engineering practices of supporting in coal roadway show that the structure failure of roadway support mainly due to the parameter selection of supporting structure is not reasonable. In fact, there are big differences between the mechanics characteristics of surrounding rock of roadway, many parameters as random variables, coupled with the influence of random factors of the physical parameters and geometric parameters of supporting structure in the course of processing, manufacturing, working, makes the deterministic model can't satisfy the need of engineering design. So it is necessary to regard all sorts of factors as random variables in reliability calculation, and consider the type of probability distribution of these variables, thus benefit for opening up a new and effective way about structural analysis and design.

*Address correspondence to this author at the College of Sciences School, Xi'an University of Science and Technology, Xi'an, Shaanxi, 710054, P.R. China; Tel: +86 13679202499; E-mail: liuming1075@163.com

\section{THE DETERMINATION OF SUPPORTING LOADS}

Effect on the arch outside loads, the main load is the pressure of surrounding rock; it is usually a main load impacting on the underground structure. The surrounding rock pressure impacted on the arch of supporting is divided into vertical pressure force and horizontal pressure force of surrounding rock [10]. For the cavity of the average level, the vertical pressure force of surrounding rock is the main force which is also the main research content of the surrounding rock pressure .In hard formation, the horizontal pressure force of surrounding rock is very small, frequently it can be neglected; In soft stratum, the horizontal pressure force of surrounding rock is larger, it must be considered in calculation.

When we determine the surrounding rock pressure, due to the influence factors of surrounding rock pressure is more and more complex, there is not completely unified calculation method so far. Platts theory is widely used in the design of underground engineering in China. To this end, this paper uses the Platts theory to determine the surrounding rock pressure. Platts theory has two basic assumptions.

1. In view of the stratum are cut by many joints and cracks which are weak structural plane, so that the surrounding rock can be treated as the loose body in a certain extent. For hard rock, Platts suggest people to compensate the ignored and actual existence of cohesive force by increasing friction coefficient between particles, the friction coefficient increased is referred to as Protodyakonov coefficient;

2. Thinking rock after excavation, due to the surrounding rock stress redistribution, the pressure arch is formed above the cavern, the weight of the rock in the arch is the surrounding rock stress impacted on the lining or the supporting; 


\subsection{The Determination of the Pressure Arch Height}

According to Platts theory, the pressure arch height is

$h_{1}=\frac{a_{1}}{f}$

$a_{1}=a+h \tan \left(45^{\circ}-\frac{\varphi}{2}\right)$

Where,

$a$--- half across of cavity;

$h$--- the cavity height;

$a_{1}$--- half across of the pressure arch;

$\varphi$--- internal friction angle of rock;

$f$--- Protodyakonov coefficient.

\subsection{The Determination of the Size of the Vertical Pressure Forswce}

The theory is that: the above of it will form a natural arch after excavation in loose medium of a certain cohesive force, and vertical pressure impacted on the supporting structure is the weight of loose rock mass within the scope of damage (natural arch), thus, the size of the vertical pressure of surrounding rock is:

$q_{1}=\gamma h_{1}$

Where,

$\gamma$--- the volume weight of surrounding rock.

\subsection{The Determination of the Size of the Horizontal Pressure Force}

The horizontal pressure force can be considered impacting on both sides of the supporting structure, the size of distributed load which impact from the top to the bottom of the underground engineering can be calculated according to the vertical pressure coefficient and lateral pressure coefficient, thus the size of the horizontal pressure of surrounding rock is:

$q_{2}=\lambda q_{1}$

Where,

$\lambda$---the lateral pressure coefficient.

When determine the outside load impacted on the supporting, we can undertake the design of roadway supporting according to the mechanical properties of supporting.

\subsection{Applied Load}

Due to the loads impacted on the roadway arch supporting structure are not the radial and tangential distributed load, with regard to such distributed load can be used by the method similar to oblique beam, the load can be treated as distribution along the axis of curved beam, and then the loads are decomposed into two parts that are the radial and tangential. Curve is more complex than linear decompo-sition, structure diagram as shown in Fig. (1), it can be exploded as follows:

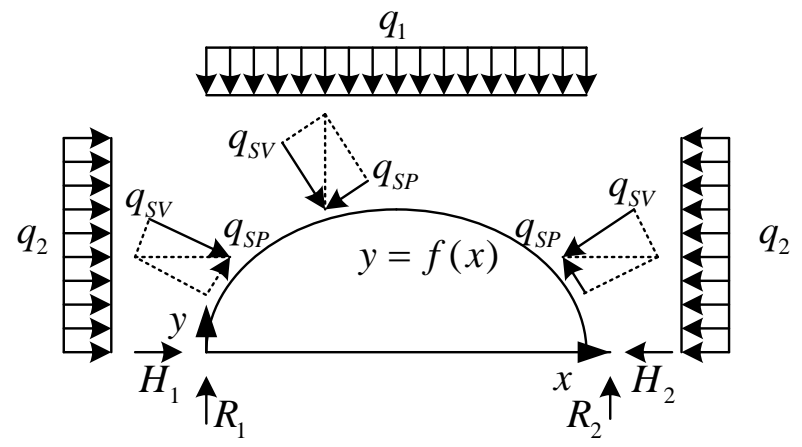

Fig. (1). Applied load.

$$
\left\{\begin{array}{l}
q_{S V}=\frac{q_{1}}{\left(1+y^{\prime 2}\right)} \\
q_{S P}=\frac{q_{1} y^{\prime}}{\left(1+y^{\prime 2}\right)}
\end{array}\right.
$$

Where,

$y^{\prime}--\mathrm{d} f(x) / \mathrm{d} x$.

When elements are divided small enough, $y^{\prime} \approx \Delta y / \Delta x$ can be used, $\Delta x$ and $\Delta y$ are the coordinate difference of two nodes in element. When the size of elements is appropriate, the results with sufficient accuracy can be calculated.

\section{RELIABILITY ANALYSIS OF SUPPORTING STRUCTURE}

Assume $x=\left(x_{1}, x_{2}, \ldots, x_{n}\right)^{T}$ as $n$ random variables that influent the supporting structure, $X$ express load parameters and structure parameters, which influent the structure function. By the strength theory of the structure, assumes that the supporting structure do not allow the structure of maximum stress $\sigma_{\max }$ more than its yield strength $\sigma_{s}$ in using process, if the stress exceeds the yield strength will be considered failure, the failure function of the roadway support structure is

$g(X)=\sigma_{s}-\sigma_{\max }$

Where,

$g(X)>0$--- structure is in a stable state;

$g(X)<0$--- structure is failure;

$g(X)=0$--- structure is in a limit state.

The reliability of the roadway support structure is the probability of $g(X)>0$.

\subsection{Monte Carlo Method}

Plug the random variables $x=\left(x_{1}, x_{2}, \ldots, x_{n}\right)^{T}$ into the failure function $g(x)$, a function value can be obtained .If 
$g(x) \leq 0$, then a failure of the implementation will be written in a computer program; If $g(x)>0$ there is nothing written in a computer program. A calculation is completed, and then generates next random number; repeat the above calculation until complete scheduled test times. At this point, the failure probability is

$$
P_{f}=P[g(x) \leq 0]=\lim _{n \rightarrow \infty} \frac{k}{n}
$$

\subsection{The Response Surface Method}

The response surface method assumes that the influence of random output variables given by the random input variables can be represented with mathematical functions. Usually function is a quadratic polynomial, the fitting function can be expressed as

$$
Z=g(X)=a+\sum_{i=1}^{n} b_{i} X_{i}+\sum_{i=1}^{j} \sum_{j=1}^{n} c_{i j} X_{i} X_{j}
$$

\subsection{The Response Surface-Monte Carlo Method}

Monte Carlo method can be combined with the finite element method to solve problems in Structural reliability analysis which the functional function can't express more briefly and directly, its calculation needs tens of thousands of times, workload is big, and time is longer than the other. Response surface - Monte Carlo method is first calculated by finite element numerical fitting a response surface instead of the unknown and the real limit state surface, then use the Monte Carlo method to sample for random variable again, $N$ times numerical calculations are made in fitting the response surface, get $N$ function value function $Z_{j}(j=1,2 \mathrm{~L}, N)$, count the number $N_{f}$ of $Z_{j}<0$ (structural failure), calculate the failure probabi-lity $p_{f}=\frac{N_{f}}{N}$. Response surface combined with Monte Carlo method reduce the workload greatly and improve efficiency for structure analysis, and overcome the shortcoming of traditional response surface for linearization error.

\section{EXAMPLE}

Take some mine roadway as an example. The width of roadway construction is $4 \mathrm{~m}$, the height of it is $2 \mathrm{~m}$. the buried depth of it is about $443 \mathrm{~m}$, the volume-weight of overburden $\gamma=24 \mathrm{kN} / \mathrm{m}^{3}$, the lateral pressure coefficient of rock (the ratio of horizontal stress and vertical stress) is 0.5 , Cohesive force of rock $\mathrm{c}=0.2 \mathrm{MPa}$, angle of internal friction $\varphi=35^{\circ}$, Protodyakonov coefficient $\mathrm{f}=1.2$. Distributed loads and elastic modulus as random variables, the mean of roof pressure $\mu_{q_{1}}=83.7 \mathrm{kN} / \mathrm{m}$, the variation coefficient of it is 0.1 , lateral pressure mean $\mu_{q_{2}}=41.9 \mathrm{kN} / \mathrm{m}$, the variation coefficient of it is 0.1 , supporting structure is the arch support, choose to use $9^{\#} \mathrm{I}$ shaped steel, the section height is $90 \mathrm{~mm}$, cross-sectional area $\mathrm{A}$ is $22.54 \mathrm{~cm}^{2}$, moment of inertia $I=I_{x}=281 \mathrm{~cm}^{4}$, the thickness of flange is $10.9 \mathrm{~mm}$, the thickness of web plate is $8 \mathrm{~mm}$, the material yield limit is $375 \mathrm{MPa}$, the mean of elasticity modulus $\mu_{E}=205 \mathrm{PPa}$, the variation coefficient of it is $0.1, \mu=0.3$.

In this paper, reliability analysis and calculation were executed by using the response surface method and Monte Carlo method hybrid simulation analysis method, cycle computing 20000 times through programming, the stress nephogram of supporting structure was obtained as shown in Fig. (2). and the random value of maximum stress of supporting structure also can be obtained as shown in Fig. (3). these stresses were sorted after each calculation, then the maximum stresses of nodes were extracted, and then put them into the defined table, and finally the histogram of maximum stress distribution of supporting structure was obtained as shown in Fig. (4). Fitting the maximum stress distribution of nodes, the probability distribution of maximum stress of supporting structure was obtained as shown in Fig. (5). In the process of reliability analysis, plug value of maximum stress distribution of the supporting structure into failure function of the roadway support



Fig. (2). The stress nephogram of supporting structure.

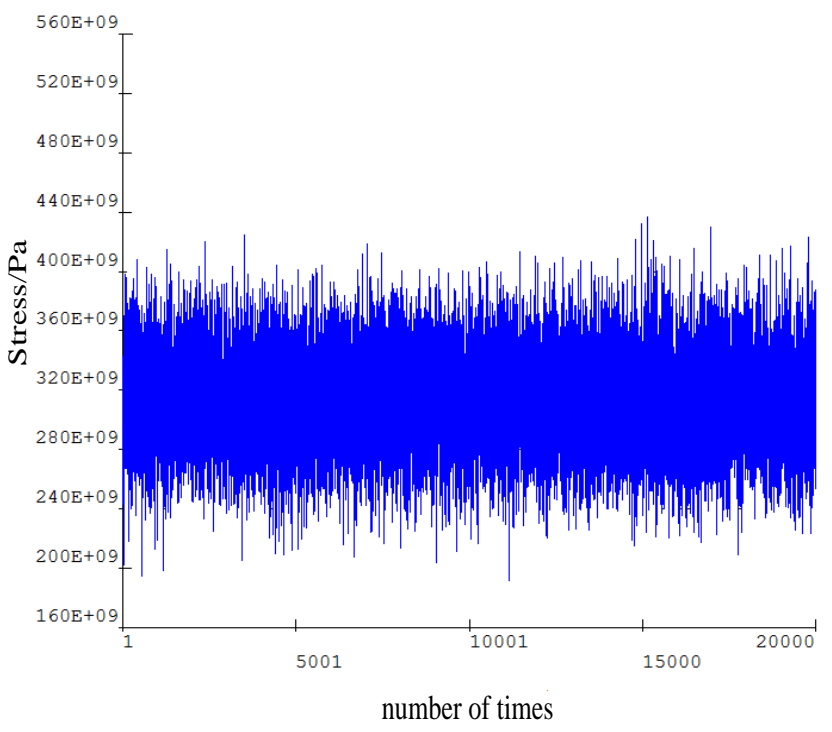

Fig. (3). The random value of maximum stress of supporting structure. 
Table 1. Probability result of response parameter maximum stress.

\begin{tabular}{|c|c|c|}
\hline Mean Value & Standard Deviation & Skewness Coefficient \\
\hline \hline $3.1370575 \mathrm{e} 8$ & $3.1368632 \mathrm{e} 7$ & $9.9433518 \mathrm{e} 5$ \\
\hline Kurtosis Coefficient & Minimum Sample Value & Maximum Sample Value \\
\hline $6.4557886 \mathrm{e} 4$ & $1.9164109 \mathrm{e} 8$ & $4.3709079 \mathrm{e} 8$ \\
\hline
\end{tabular}

structure. Finally a probability of $g(x)>0$ was obtained through statistics. The probability can be seen that maximum stress is smaller than $375 \mathrm{MPa}$ is $9.74630 \mathrm{e}-001$ by the numerical simulation results, its lower bound is $9.72389 \mathrm{e}-$ 001, and its upper bound is 9.76748e-001.The confidence bounds are evaluated with a confidence level of $95.000 \%$. The probability is interpolated between: maximum stress = $3.7499415 \mathrm{e}+008$ which has rank 19493 out of 20000 samples, maximum stress $=3.7501739 \mathrm{e}+008$ which has rank 19494 out of 20000 samples. The probability result of response parameter maximum stress as shown in Table $\mathbf{1 .}$ We can see the reliability of arched roadway supporting structure is $97.463 \%$ by the numerical results.



Fig. (4). The histogram of maximum stress distribution of supporting structure.

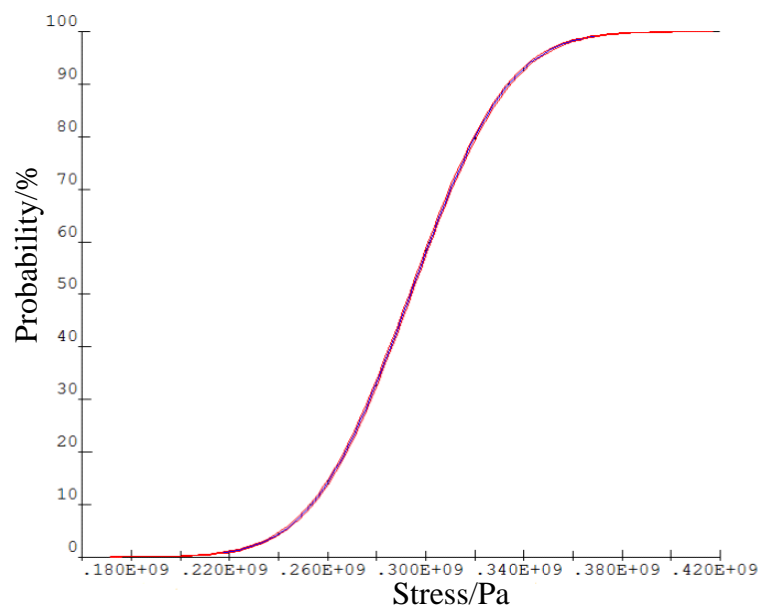

Fig. (5). The probability distribution of maximum stress of supporting structure.

\section{CONCLUSION}

(1) The paper based on the arched retaining structure of soft rock roadway, established the finite element model of arched roadway support structures with stochastic parameters, through the analysis that the load and the supporting structure physical parameters and geometric parameters of structural materials can be used as the random variables of reliability analysis, and the mean and coefficient of variation of random variables are given. then use the mean and variable coefficient of random variables to make the finite element pre-processing. At last, the reliability indexes are presented through the response surface method and Monte Carlo method for reliability analysis.

(2) Through the numerical analysis, it is observed that the randomness of the supporting structure parameters and the randomness of loads have a certain influence on reliability of arch supporting structure, we should attach importance to it.

\section{ABOUT THE AUTHORS}

\section{First Author}

Liu Ming, University lecturer of Xi'an University of Science and Technology, Ph.D. The author's major is Rock mechanics and engineering. 10 papers received by EI or CSCD had been published.

\section{Second Author}

WU Yongping, University professor of Xi'an University of Science and Technology, Ph.D. The author's major is mining engineering. More than 40 papers received by EI or CSCD had been published.

\section{Third Author}

Qiao Xinzhou, University associate professor of Xi'an University of Science and Technology, Ph.D. The author's major is mining machinery.

\section{CONFLICT OF INTEREST}

The author confirms that this article content has no conflict of interest.

\section{ACKNOWLEDGEMENTS}

This work was financially supported by China Postdoctoral Science Foundation (2014M562519XB), the Xi'an University of Science and Technology Doctor Star-up Foundation (2014QDJ072)and Scientific research cultivating fund (201348). 


\section{REFERENCES}

[1] A.M. Freudenthal "The safety of structures", Transactions of the American Society of Civil Engineers, vol. 112, pp. 125-159 Jan, 1947.

[2] A.M. Freudenthal "Safety and the probability of structural failure", Transactions of the American Society of Civil Engineers, vol. 121, pp. 1337-1397, 1956.

[3] A.M, Hasofer N.C. Lind "Exact and invariant second-moment code format", Journal of the Engineering Mechanics Division, vol. 100, pp. 111-121, Jan/Feb, 1974.

[4] R. Rackwitz B. Fiessler "Structural reliability under combined random load sequences", Computers and Structures, vol. 9, pp. 489-494, 1978.
P. Thoft-Chritsensen J.D. Sorensen "Reliability of structural systems with correlate elements", Applied Mathematical Modeling, vol. 6, pp. 171-178, 1982.

6] M. Hohenbichler R. Rackwitz "First-order concepts in system reliability”, Structual Safety, vol. 1, pp. 177-188, 1983.

] , Ditlevsen "Taylor expansion of series system reliability", Jounal of Engineering Mechanics, vol. 110, pp. 293-307, 1984.

8] O. Ditlevsen, R. Melchers, H. Gluver "General mutlidimention probalility integraion by directional simulation", Computer \& Structure vol. 36, pp. 355-368, 1990.

9] Y.S. Feng "The computation of failure probability for nonlinear safety", Reliability Engineering and System, vol. 27, pp. 323-331, 1990.

[10] R.E. Melchers "Importance sampling in structural system", Structure Safety, vol. 6, pp. 3-10, 1989.

(C) Ming et al.; Licensee Bentham Open.

This is an open access article licensed under the terms of the Creative Commons Attribution Non-Commercial License (http://creativecommons.org/licenses/ by-nc/3.0/) which permits unrestricted, non-commercial use, distribution and reproduction in any medium, provided the work is properly cited. 